\title{
An upgraded binary bat algorithm approach for optimal allocation of PMUs in power system with complete observability
}

\author{
M. Ravindra*, R. Srinivasa Rao \\ Department of Electrical and Electronics Engineering, Jawaharlal Nehru Technological University Kakinada, India
}

\section{A R T I C LE IN F O}

\section{Article history:}

Received 19 June 2017

Received in revised form

14 August 2017

Accepted 15 August 2017

\section{Keywords:}

Bat algorithm

Branches

Observability

PMUs

Synchrophasors

\begin{abstract}
A B S T R A C T
This paper presents an Upgraded Binary Bat Algorithm (UBBA) approach for optimal allocation of Phasor Measuring Units (PMUs) in power system network with complete observability. In power system grid network, allocations of Phasor Measuring Units (PMUs) at buses differ in cost on the grounds that the number of branches associated with every bus of the network varies. The weight of all the branches considered in the optimization process to assess the cost for allocation of PMUs. The Bus Redundancy Index (BRI) at each bus is taken in to consideration to estimate the performance of complete observability of the network. UBBA developed in such ways that complete observability of system is obtained with a minimum cost. The proposed UBBA is programmed in MATLAB and simulated on IEEE 14-, 24-, $30-$, and 57 - bus systems to obtain optimal allocation of PMUs. In order to describe the advantage of proposed method, its simulation results are analyzed and compared with different strategies available in the literature.
\end{abstract}

(C) 2017 The Authors. Published by IASE. This is an open access article under the CC BY-NC-ND license (http://creativecommons.org/licenses/by-nc-nd/4.0/).

\section{Introduction}

The present state estimation process of wide area monitoring systems (WAMS) with the data obtained from Supervisory Control and Data Acquisition (SCADA) is lagging measurement and estimation accuracy. With the introduction of synchrophasor measurements into the power system, the subject of WAMS and state estimation is receiving focus from researchers in this area. PMUs are devices which measure phasors associated with voltage and current of a bus and synchronize the measurements with the time signal received from the Global position system (GPS) (Phadke and Thorp, 2008; Phadke et al., 1986). In power system network, allocation of PMU at every bus of the network is infeasible which leads to high cost. PMUs should be allocated at buses in such way that system does not lose observability. For allocation of PMU, a bus incident with more branches increases PMU installation cost at that bus.

So to minimize installation cost, PMUs should be allocated at a bus with a redundant number of branches without losing observability. For PMU placement, many authors use integer linear

\footnotetext{
* Corresponding Author.

Email Address: ravieeejntu@gmail.com (M. Ravindra) https://doi.org/10.21833/ijaas.2017.010.006

2313-626X/C) 2017 The Authors. Published by IASE.

This is an open access article under the CC BY-NC-ND license

(http://creativecommons.org/licenses/by-nc-nd/4.0/)
}

programming (ILP) (Gou, 2008). The convergence of this method takes a longtime for large power systems. In past 10 years many researchers presented algorithms like non-dominated genetic algorithm (Milosevic and Begovic, 2003) and immunity genetic algorithms (Aminifar et al., 2009) a novel genetic algorithm (Muller and Castro, 2016) is proposed for PMU placement considering security issues and observability. Observability in case of line outage or PMU loss is preserved in this procedure. These genetic algorithms proposed till now use many functions to solve the problem and converge slowly leading to inadequate solutions. In problem proposed by Korres et al. (2015), observability of system is obtained through finding rank of the Jacobian matrix numerically. With introduction of creative new optimization methods like BPSO (Ahmadi et al., 2011), CRO (Xu et al., 2013) ACA (Bian and Qiu, 2006), the optimization of PMU placement becomes easier with faster convergence rate. Yang (2010) proposed a meta-heuristic bat algorithm and compared with existing PSO, Firefly, and HSA. Mirjalili et al. (2014) proposed Binary Bat Algorithm (BBA) considering binary variables $(0,1)$. For optimal placement of PMU problem, the initialization of solution vector using this method does not solve the problem.

This algorithm is upgraded with changing the initialization of the algorithm in which initialization of memory is considered similar to HSA proposed by Rao et al. (2011). The proposed algorithm is 
modeled using HSA and BBA for optimal allocation of PMUs in the network.

This paper presents UBBA approach for optimal allocation of PMUs considering cost as criteria for minimization. Weight of every branch is considered for modeling the cost constraints in the optimization problem. The redundancy at every bus is considered to achieve complete observability of system network.

The remaining part of paper is organized as follows: Section 2 describes problem formulation; Section 3 deals with proposed UBBA; Section 4 presents the application of PMU placement problem with proposed method; Section 5 results with discussion of the problem with MATLAB simulation results and Section 6 concludes the problem.

\section{Problem formulation}

The main objective function is formulated to minimize the cost function with minimum number of PMUs forming complete observability of system (Eqs.1 and 2):

$\operatorname{Min} \sum_{j=1}^{N} C_{j} x_{j}$

Subject to $\quad A X \geq B$

where $C_{j}$ is defined as cost coefficient of PMU installed at bus ' $j$ ' in the network, $X=$ $\left[\begin{array}{lllll}x_{1} & x_{2} & x_{3} & \ldots & \ldots \\ x_{n}\end{array}\right]^{T}$ is a binary variable marix in which $x_{j}$ is binary decision variable, $B$ is an array of observability constraints which can be written as $\left[\begin{array}{llll}1 & 1 & 1 & \ldots \ldots . .1\end{array}\right]_{n \times 1}^{T}$ and $A$ is bus incidence matrix which is defined as

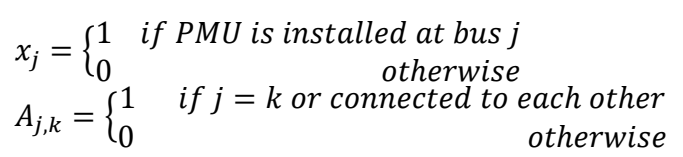

Minimum cost for installation of PMU at buses is selected based on weight of all branches connected to a bus. The weight of every branch is equal, but the more branches connected to bus the more the installation cost. So to decrease cost of installation, the optimal numbers of PMUs for selected buses with limited branches are considered for installation.

\section{Modeling of UBBA}

Bat algorithm (BA) is a creative optimization process developed by Yang (2010). The BA is centered on the behavior of bats with changing loudness and pulse rates of emission (Mirjalili et al., 2014). Bats have enormously refined sense of hearing. They emit sounds that bounce off particles in their path transmitting echoes back to bats. From the echoes, the bats can investigate size of objects, how a long way away they are, how fast they are touring and even their texture, all in a break up sounds. Rules used by Yang (2010).

- Bats make use of echolocation to find distance
- Bats fly indiscriminately with velocity $v_{j}$ at position $X_{j}$ with frequency $f_{\min }$ and loudness $L_{0}$ to hunt for prey. Bats are capable of changing wavelength and pulse emission rate $r \in(0,1)$ depending upon the target.

- Loudness of bat varies in different ways, but it is considered in our problem we assume that loudness vary from high $L_{0}$ to low constant value $L_{\text {min }}$

\subsection{Problem initialization and parameters}

The binary optimization problem can be derived as (Eqs. 3 and 4)

$\operatorname{Min} F(x)$

Subjected to $x_{j} \in X_{j} \quad j=1,2, \ldots . N$

where $F(x)$ is objective function. $x$ is binary variable or position vector and $X$ is vector of $\mathrm{N}$ number of decision or binary variables.

Parameters considered for BBA are velocity vector $v_{j}$ frequency vector $f_{j}$, pulse emission rates $r_{j}$ and the loudness $L_{j}$, which are updated during iterations.

\subsection{Initialization of binary bat memory}

The solution vector assumed as a row vector $\left[x_{1} x_{2} x_{3} \ldots \ldots x_{n}\right]$ of n-bus, generated with 1 random decision variables $(0,1)$. The row vectors of population size which satisfies the subjected constraints are arranged in a matrix initializing bat memory as follows (Eq. 5):

$$
B M=\left[\begin{array}{lllcc}
x_{1}^{1} & x_{2}^{1} & \ldots & x_{n-1}^{1} & x_{n}^{1} \\
x_{1}^{2} & x_{2}^{2} & \ldots & x_{n-1}^{2} & x_{n}^{2} \\
\vdots & \vdots & \vdots & \vdots & \vdots \\
\vdots & \vdots & \vdots & \vdots & \vdots \\
x_{1}^{p-1} & x_{2}^{p-1} & \ldots & x_{n-1}^{p-1} & x_{n-1}^{p-1} \\
x_{1}^{p} & x_{2}^{p} & \ldots & x_{n-1}^{p} & x_{n}^{p}
\end{array}\right]
$$

\subsection{Fitness function with binary update}

The objective function value is considered as fitness value that satisfies the constraints. The weight vector is used to define cost value depending on factor of installation and manufacture criteria.

$\operatorname{fitness}(x)=\sum_{j=1}^{n} C_{j} x_{j}$

where $C_{j}$ is weight matrix in the form of a diagonal matrix, normally considered as a diagonal unit vector or weight can be increased or decreased i.e., varies between 0 and 2 . In continuous domain of BA, the suggested bats can proceed within domain utilizing position and velocity vectors. The strategy of updating positions can applied for bats by addition velocities to positions using (8) however updating position is different in binary space (Eqs. 79)

$f_{j}=f_{\text {min }}+\left(f_{\max }-f_{\text {min }}\right) \beta$ 
$x_{j}(t+1)=x_{j}(t)+v_{j}(t$

$v_{j}(t+1)=v_{j}(t)+\left(x_{j}(t)-g_{\text {best }}\right) f_{j}$

where $\beta$ is considered as a random number between $[0,1]$ and $g_{\text {best }}$ is best solution obtained, $f_{j}$ is frequency of the $j^{\text {th }}$ bat updated with iterations.

To make similarity of velocity values to probability values, generally transfer function range considered is [0 1].The function should produce high probability of position change for large velocity change and the small probability of change position for small velocity change. The return transfer function value should increase with the velocity rise and decrease as velocity decrease. With this concept, transfer function is able to bring the similarity and map the continuous search space to a binary search space. The V-shaped transfer function is formulated as follows (Eqs. 10 and 11):

$T\left(v_{j}^{k}(t)=\left|\frac{2}{\pi} \arctan \left(\frac{\pi}{2} v_{j}^{k}(t)\right)\right|\right.$

$x_{j}^{k}(t+1)=\left\{\begin{array}{lr}\left(x_{j}^{k}(t)\right)^{-1} & \text { if rand }<T\left(v_{j}^{k}(t+1)\right) \\ x_{j}^{k}(t) & \text { rand } \geq T\left(v_{j}^{k}(t+1)\right)\end{array}\right.$

where $x_{j}^{k}(t)$ is position and $v_{j}^{k}$ is velocity of $j^{t h}$ article at iteration ' $t$ ' in $k^{\text {th }}$ dimension

For local search method once the most effective solution is chosen from current global best solution, a new solution is generated for every bat from the procedure of random walk as follows:

$x_{\text {new }}=x_{\text {old }}+\varepsilon L^{T}$

where $\epsilon \in[1,1]$ is random number, $L$ is average or mean of loudness emitted from bats considered.

\subsection{Loudness and pulse emission}

UBBA is balancing conception of HSA and local search method during which the balancing is controlled by loudness $(L)$ and pulse emission $r$. The $L_{j}$ and $r_{j}$ are updated with iterations as shown.

$L_{j}(t+1)=\alpha L_{j}(t)$

$r_{j}(t+1)=r_{j}(0)[1-\exp (-\gamma t)]$

where $\alpha$ and $\gamma$ are constants, $\alpha$ is identical to the cooling factor in Kirkpatrick et al. (1983), for any $0<$ $\alpha<1$ and $\gamma>0$ we have $A_{j}(t) \rightarrow 0, r_{j}(t) \rightarrow$ $r_{j}(0)$ as $t \rightarrow \infty$.

Both loudness and pulse emissions are updated once the new solution is improved and moving towards most effective solution.

\section{Optimal allocation of PMU using an UBBA}

The initial requirements for optimization are bus incidence matrix $A$ which describes the connectivity of the buses in network considered, B as vector observability constraints.

Solution vector $[X]$ considered as row vector $X=$ $\left[\begin{array}{lllll}x_{1} & x_{2} & x_{3} & \ldots & \ldots \\ x_{n}\end{array}\right]^{T}$ for location of PMUs. Binary bat memory is organized with $\mathrm{P}$ population row vectors of n-bus system.

The parameters considered for optimization are shown in Table 1 . Observability is checked at the stage of initializing memory of bat and at the end of the algorithm to obtain complete observability of system network. During initialization of the problem each row in the matrix is considered as one population number, in which each row is decision variable matrix to allocate PMU. A random row matrix of n-number of buses, $\mathrm{p}$ - number of population is considered initially which is subjected to observability condition. While considering cost analysis in the problem the weight $\left(C_{j}\right)$ for each PMU is considered as $1 \mathrm{p} . \mathrm{u}$ value and is represented as a diagonal matrix. In previous papers published cited in literature no author considered the weight of each branch. In this work, the addition of branch weight which is considered as 0.1p.u for every branch connected to bus is added to PMU weight.

\begin{tabular}{cc} 
Table 1: UBBA parameters \\
\hline Population & 30 \\
\hline Loudness & 0.02 \\
Pulse rate & 0.1 \\
Maximum iterations & 300 \\
\hline
\end{tabular}

The flowchart of UBBA approach for optimal allocation of PMUs in the network is shown in Fig. 1.

\section{Results and analysis}

To allocate PMUs and check complete observability, four different test cases such as 14-, 24-, 30- and 57-bus test systems are considered to analyze applicability of proposed UBBA. The optimal PMU allocation problem with UBBA is programmed in MATLAB and it is run on Intel(R) core(TM), an i3 processor at $2.20 \mathrm{GHz}$ with $4 \mathrm{~GB}$ of RAM. UBBA is modeled for allocation of PMUs considering branch weight and redundancy of network.

The single line diagrams of 14-and 24-bus system are shown in Figs. 2 and 3. Total branch weight at every bus of IEEE 14- bus 24-bus systems that is added is shown in Figs. 4 and 5.

Table 2 shows, minimum number of PMUs and installation locations considering cost analysis based on weight of the branches. The allocation places are at the buses with less branch weight which reduces installation cost of the PMUs.

The convergence characteristics of cost function of 14-bus, 24-bus, 30-bus and 57-bus are shown in Figs. 6, 7, 8, and 9.

From Figs. 6, 7, 8, and 9 it is observed that UBBA converges faster within a few iterations. Installation cost of PMUs at different buses with the different number of branches is more, so in order to reduce cost of installation, the total branch weight of the bus with limited number of branches is selected for installing the PMU.

For 14-bus system, optimal location of PMUs are $2,8,10$, and 13 and their branch weights are $(0.4+0.1+0.2+0.3)+4$ PMUs weight, which is equal to 
5 p.u in total and similarly to 24 bus system the branch weight is $(0.3+0.2+0.1+0.5+0.5+0.2+0.3+0.2)$ +8 PMUs weight, which is 10.3 p.u. in total.

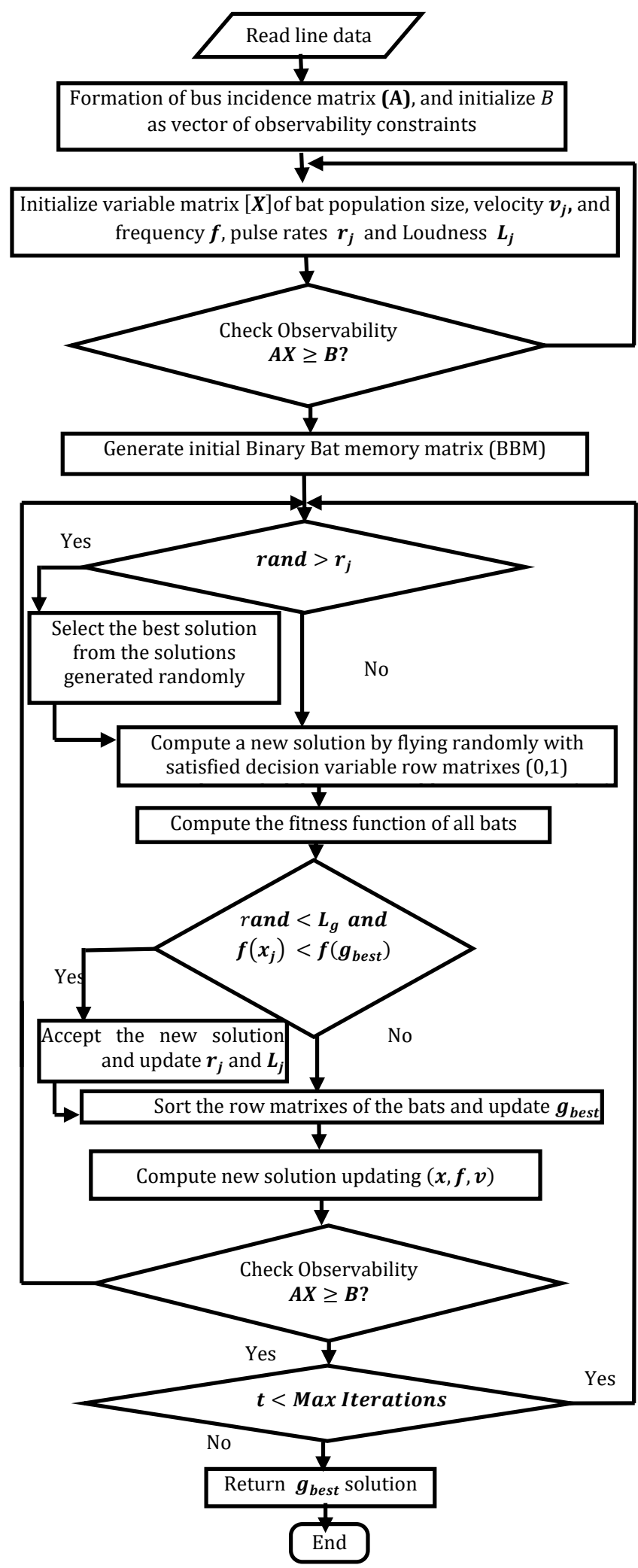

Fig. 1: Flow chart of optimal allocation of PMUs considering UBBA approach

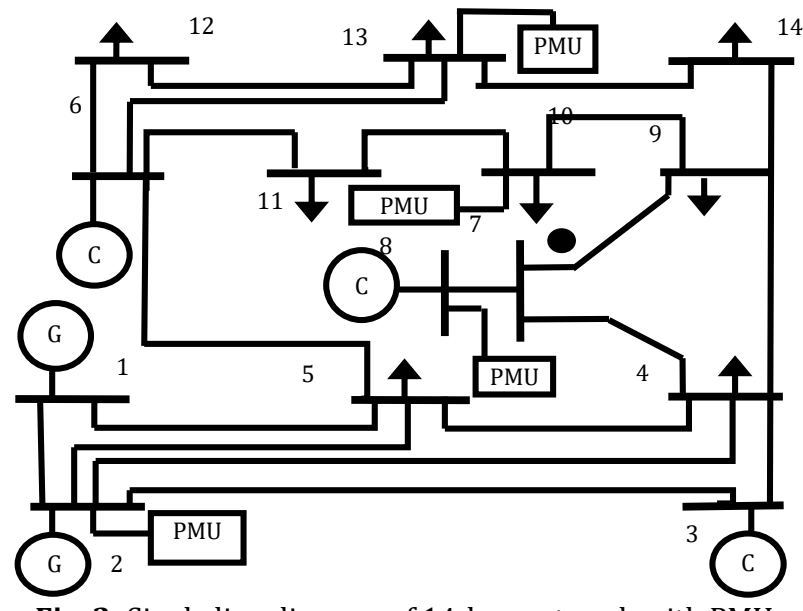

Fig. 2: Single line diagram of 14-bus network with PMU

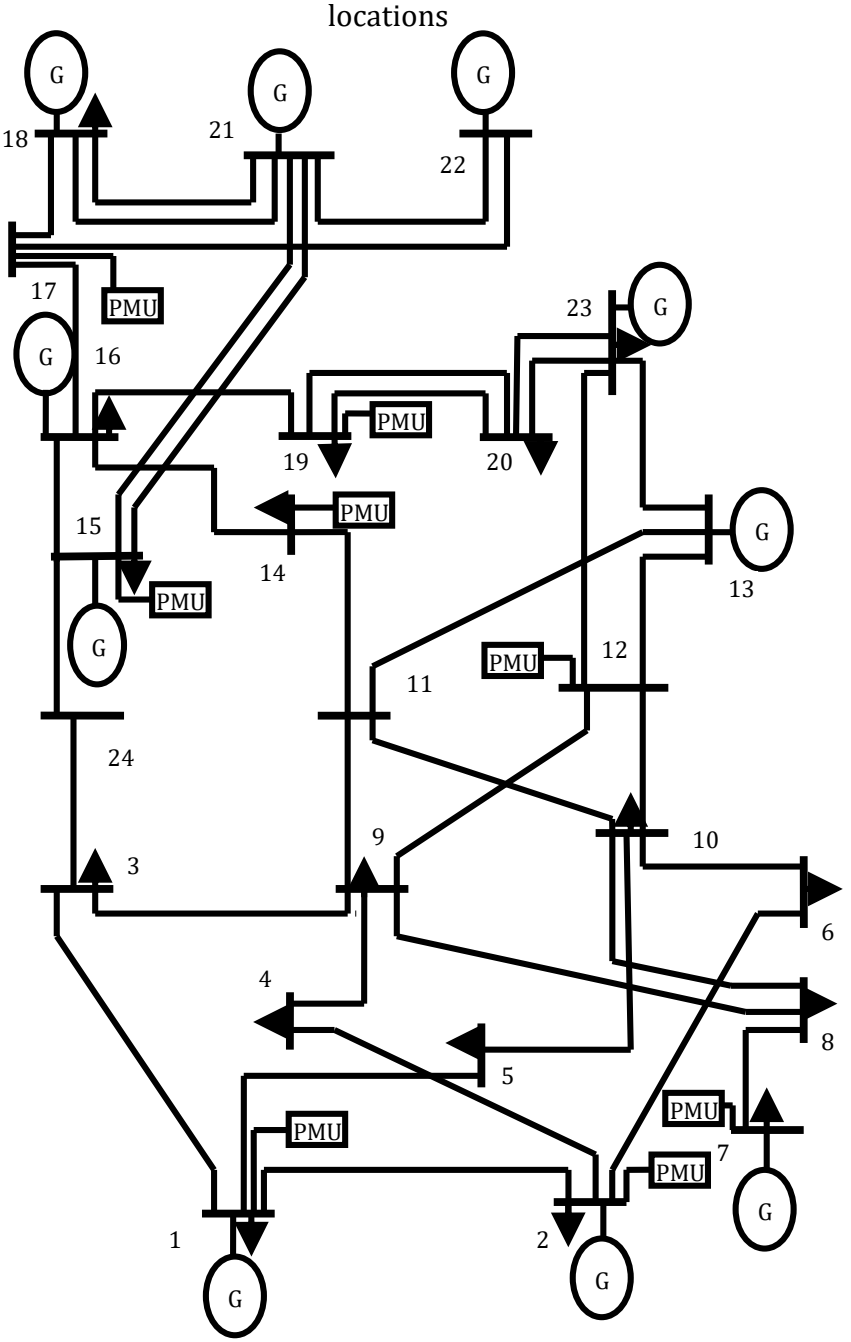

Fig. 3: Single line diagram of 24-bus network with PMU locations

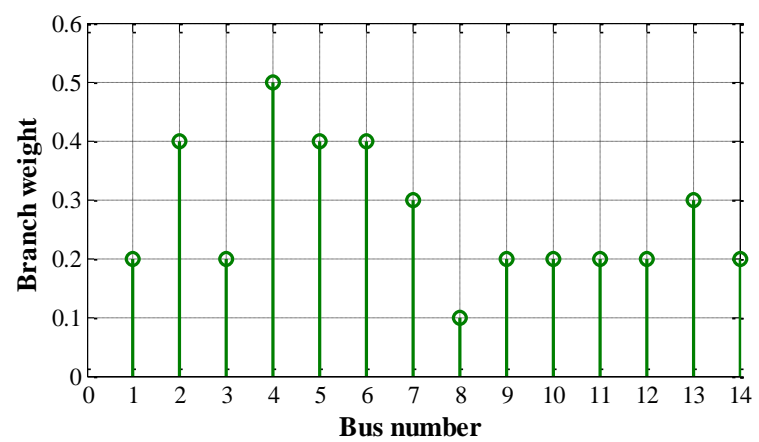

Fig. 4: Branch weight of 14 bus system 


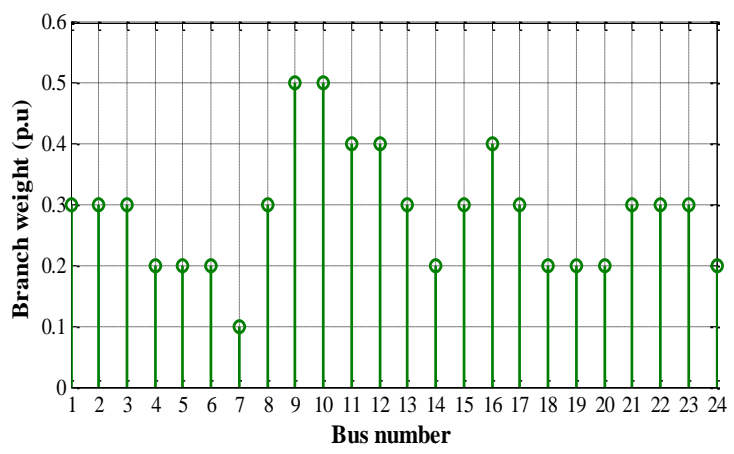

Fig. 5: Branch weight of 24 bus system

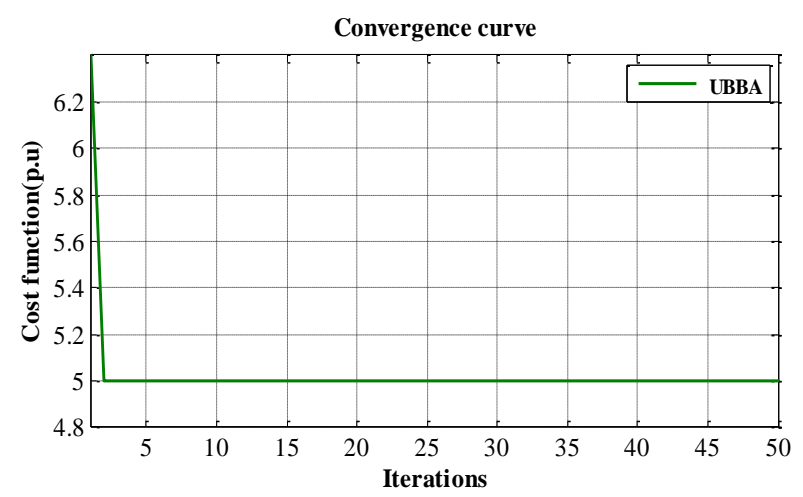

Fig. 6: Cost function for 14-bus system

Table 2: PMU locations with UBBA approach

\begin{tabular}{ccc}
\hline IEEE Test case Systems & No. of PMUs & PMU locations \\
14 bus & 4 & $2,8,10,13$ \\
24 bus & 8 & $1,2,7,12,14,15,17,19$ \\
30 bus & 10 & $1,5,8,9,10,12,18,23,25,30$ \\
57 bus & 19 & $1,4,9,10,15,20,23,27,29,30,32,36,38,39,41,46,49,53,56$ \\
\hline
\end{tabular}

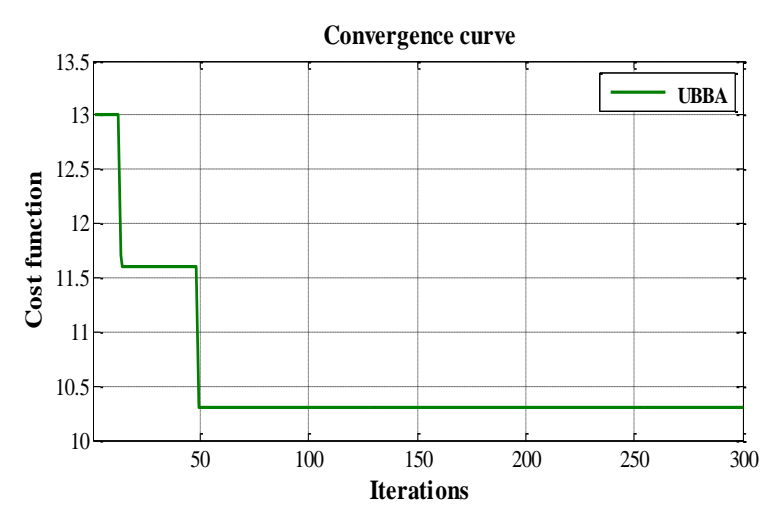

Fig. 7: Cost function for 24 bus system

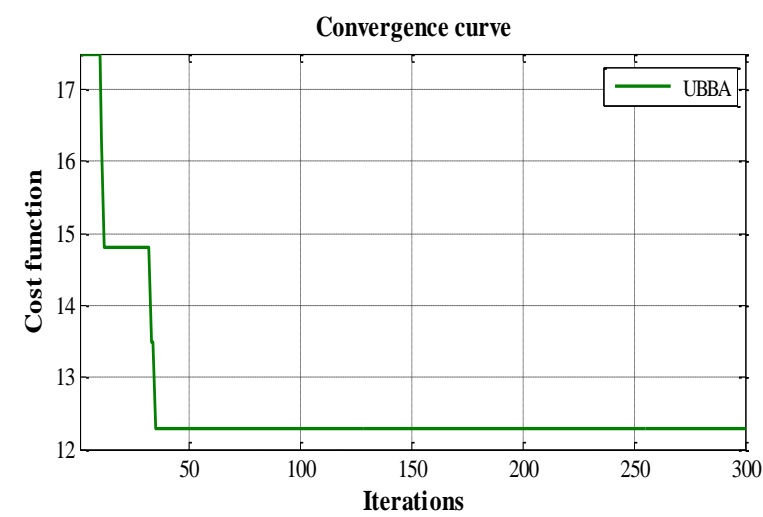

Fig. 8: Cost function for 30 bus system

Consider 14-bus system, for which (Bus Redundancy Index) BRI is computed at every bus to estimate number of times bus is observed by PMU to achieve full observability of bus network. BRI of the network can be formulated as

$B R I=A X$ where $X=\left[\begin{array}{lllll}x_{1} & x_{2} & x_{3} & \ldots & \ldots \\ x_{n}\end{array}\right]^{T}$ is a binary variable matrix in which $x_{j}$ is binary decision variable and $A$ is incidence matrix.

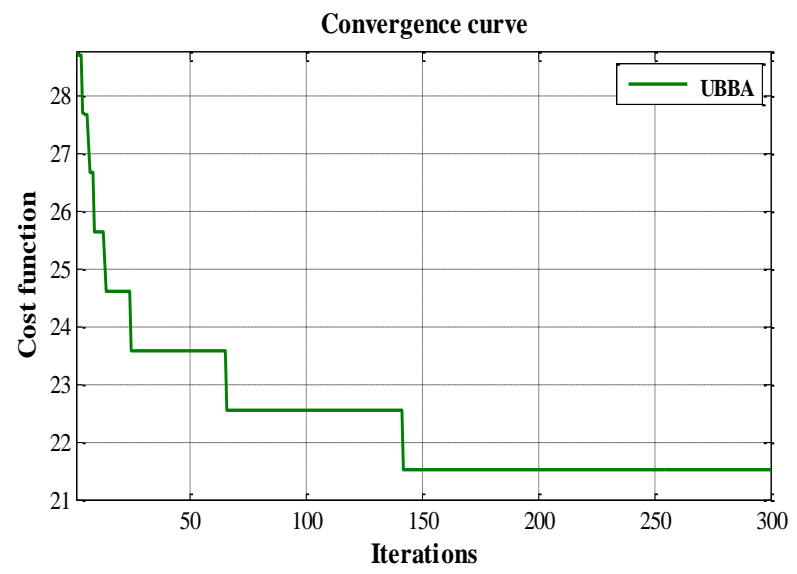

Fig. 9: Cost function for 57 bus system

Performance of UBBA approach for optimal allocation of PMUs can be computed with BRI for different IEEE test case systems as foolows

Table 3, 4, 5 and 6 shows redundancy index at every bus of different IEEE test case systems. The network is said to be completely observable if every bus of network is observed by PMU at least one time.

In this paper, UBBA is formulated to consider the bus with a limited number of branch weights for optimal allocation of PMUs

Table 3: Bus redundancy index

\begin{tabular}{cccccccc}
\hline Bus. No & 1 & 2 & 3 & 4 & 5 & 6 & 7 \\
BRI & 1 & 1 & 1 & 1 & 1 & 1 & 1 \\
Bus. No & 8 & 9 & 10 & 11 & 12 & 13 & 14 \\
BRI & 1 & 1 & 1 & 1 & 1 & 1 & 1 \\
\hline
\end{tabular}

Table 4: Bus redundancy index of 24-bus system

\begin{tabular}{cccccccccccccc}
\multicolumn{1}{c}{ Table 4: } & \multicolumn{10}{c}{ (1) redundancy index of 24 -bus system } \\
\hline Bus. No & 1 & 2 & 3 & 4 & 5 & 6 & 7 & 8 & 9 & 10 & 11 & 12 \\
BRI & 2 & 2 & 1 & 1 & 1 & 1 & 1 & 1 & 1 & 1 & 1 & 1 \\
Bus. No & 13 & 14 & 15 & 16 & 17 & 18 & 19 & 20 & 21 & 22 & 23 & 24 \\
BRI & 1 & 1 & 1 & 4 & 1 & 1 & 1 & 1 & 1 & 1 & 1 & 1 \\
\hline
\end{tabular}

Table 5: Bus redundancy index of 30-bus system

\begin{tabular}{|c|c|c|c|c|c|c|c|c|c|c|c|c|c|c|c|}
\hline Bus. No & 1 & 2 & 3 & 4 & 5 & 6 & 7 & 8 & 9 & 10 & 11 & 12 & 13 & 14 & 15 \\
\hline BRI & 1 & 2 & 1 & 1 & 1 & 3 & 1 & 1 & 2 & 2 & 1 & 1 & 1 & 1 & 3 \\
\hline Bus. No & 16 & 17 & 18 & 19 & 20 & 21 & 22 & 23 & 24 & 25 & 26 & 27 & 28 & 29 & 30 \\
\hline BRI & 1 & 1 & 1 & 1 & 1 & 1 & 1 & 1 & 2 & 1 & 1 & 2 & 1 & 1 & 1 \\
\hline
\end{tabular}


Table 6: Bus redundancy index of 57-bus system

\begin{tabular}{cccccccccccccccccccccccccccccccc}
\hline Bus No & 1 & 2 & 3 & 4 & 5 & 6 & 7 & 8 & 9 & 10 & 11 & 12 & 13 & 14 & 15 & 16 & 17 & 18 & 19 & 20 & 21 & 22 & 23 & 24 & 25 & 26 & 27 & 28 & 29 & 30 \\
BRI & 2 & 1 & 2 & 1 & 1 & 1 & 1 & 1 & 2 & 2 & 2 & 2 & 3 & 2 & 2 & 1 & 1 & 1 & 1 & 1 & 1 & 2 & 1 & 1 & 1 & 1 & 1 & 2 & 1 & 1
\end{tabular}

Bus. No $31 \quad 32 \quad 33 \quad 34 \quad 35 \quad 36 \quad 37 \quad 38 \quad 39 \quad 40 \quad 41 \quad 42 \quad 43 \quad 44 \quad 45 \quad 46 \quad 47 \quad 48 \quad 49 \quad 50 \quad 51 \quad 52 \quad 53 \quad 54 \quad 55 \quad 56 \quad 57$

$\begin{array}{lllllllllllllllllllllllllllll}\text { BRI } & 2 & 1 & 1 & 1 & 1 & 1 & 3 & 2 & 1 & 2 & 2 & 2 & 1 & 1 & 1 & 1 & 1 & 2 & 2 & 1 & 1 & 2 & 1 & 1 & 1 & 2 & 2\end{array}$

PMU locations and their installation cost values are shown in Table 7 for a 14-bus system. From the table, it is observed that for 4 PMU locations the cost value differs, when buses are changed. This is because different buses are connected with different number of branches which adds the weight and increases, cost of installation. Table 8 and 9 shows comparison of cost function values of proposed method for 24 -bus system and 30-bus system with different methods. Time consumed by UBBA for different test case systems is shown in Table 10.

Table 7: Comparison of cost with PMU locations for 14- bus system

\begin{tabular}{|c|c|c|}
\hline Methods & $\begin{array}{l}\text { Installation Cost of } \\
\text { PMU [p.u] }\end{array}$ & $\begin{array}{c}\text { PMU } \\
\text { Locations }\end{array}$ \\
\hline $\begin{array}{l}\text { BPSO (Ahmadi et al., 2011), ACA (Bian and Qiu, 2006), Exhaustive search (Chakrabarti et al., 2009), } \\
\text { BGO (Jamuna and Swarup, 2012), GA (Bedekar et al., 2011), CRO (Xu et al., 2013) }\end{array}$ & 5.5 & $2,6,7,9$ \\
\hline BGO (Jamuna and Swarup, 2012), ACA (Bian and Qiu, 2006) & 5.2 & $2,7,10,13$ \\
\hline BILP (Abbasy and Ismail,2009), ACA (Bian and Qiu, 2006) & 5.3 & $2,6,7,9$ \\
\hline UBBA & 5.3 & $2,6,7,9$ \\
\hline UBBA & 5.2 & $2,7,10,13$ \\
\hline UBBA & 5.1 & $2,6,8,9$ \\
\hline UBBA & 5 & $2,8,10,13$ \\
\hline
\end{tabular}

Table 8: Comparison of cost with PMU locations for 24- bus system

\begin{tabular}{ccc}
\hline \multicolumn{2}{c}{ Methods } & $\begin{array}{c}\text { Installation Cost of PMU } \\
\text { [p.u] }\end{array}$ \\
\hline BILP (Abbasy and Ismail, 2009), BPSO (Ahmadi et al., 2011) & 10.5 & $1,2,8,11,16,21,23,24$ \\
BGO (Jamuna and Swarup, 2012), ACA (Bian and Qiu, 2006) & 10.4 & $2,5,8,11,16,21,23,24$ \\
UBBA & 10.5 & $1,2,8,11,16,21,23,24$ \\
UBBA & 10.5 & $2,5,8,9,16,21,23,24$ \\
UBBA & 10.4 & $2,5,8,11,16,21,23,24$ \\
UBBA & 10.3 & $3,4,7,10,11,14,17,18$ \\
\hline
\end{tabular}

Table 9: Comparison of cost with PMU locations for 30- bus system

\begin{tabular}{ccc}
\hline Methods & Installation Cost of PMUs [p.u] & PMU Locations \\
Integer quadratic programming (Chakrabarti and Kyriakides, 2008) & 13.5 & $2,4,6,9,10,12,15,19,25,27$ \\
Exhaustive search (Chakrabarti et al., 2009) & 13.3 & $1,2,6,9,10,12,15,19,25,27$ \\
CRO (Xu et al., 2013) & 13 & $2,4,6,9,10,12,19,23,25,26$ \\
BGO (Jamuna and Swarup, 2012) & 12.9 & $1,5,6,9,10,12,15,18,25,29$ \\
UBBA & 12.8 & $3,5,10,11,12,18,24,25,27,28$ \\
UBBA & 12.7 & $3,5,6,10,11,12,19,23,25,30$ \\
UBBA & 12.4 & $1,5,9,10,12,19,23,26,28,30$ \\
UBBA & 12.3 & $1,5,8,9,10,12,18,23,25,30$ \\
\hline
\end{tabular}

Table 10: Time consumed for test case systems

\begin{tabular}{cc}
\hline IEEE Test cases & Time(s) \\
\hline 14 bus system & 1.572 \\
24 bus system & 2.390 \\
30 bus system & 3.075 \\
57 bus system & 11.388 \\
\hline
\end{tabular}

\section{Conclusion}

A new Upgraded Binary Bat algorithm approach is presented for optimization of PMU locations by decision variable vector matrix in binary form. The optimal PMU allocation in the power system with complete observability is achieved. Installation cost of PMUs at a bus is considered by adding weight of branches incident on the buses. Minimum cost for installation of PMUs is achieved in optimizing the bus location by considering total weight of the bus. Bus Redundancy Index (BRI) shows bus redundancy at every bus forming complete observable network. MATLAB simulation results show the efficacy of proposed method with minimum number of PMUs forming complete observable.

\section{References}

Abbasy NH and Ismail HM (2009). A unified approach for the optimal PMU location for power system state estimation. IEEE Transactions on Power Systems, 24(2): 806-813.

Ahmadi A, Alinejad-Beromi Y, and Moradi M (2011). Optimal PMU placement for power system observability using binary particle swarm optimization and considering measurement redundancy. Expert Systems with Applications, 38(6):72632769.

Aminifar F, Lucas C, Khodaei A, and Fotuhi-Firuzabad M (2009). Optimal placement of phasor measurement units using immunity genetic algorithm. IEEE Transactions on Power Delivery, 24(3):1014-1020.

Bedekar PP, Bhide SR, and Kale VS (2011). Optimum PMU placement considering one line/one PMU outage and maximum redundancy using Genetic algorithm. In the $8^{\text {th }}$ International Conference on Electrical Engineering/ Electronics, Computer, Telecommunications and Information 
Technology, IEEE, Khon Kaen, Thailand: 688-691. https://doi.org/10.1109/ECTICON.2011.5947933

Bian X and Qiu J (2006). Adaptive clonal algorithm and its application for optimal PMU placement. In the International Conference on Communications, Circuits and Systems Proceedings, IEEE, Guilin, China, 3: 2102-2106. https://doi.org/10.1109/ICCCAS.2006.284913

Chakrabarti S and Kyriakides E (2008). Optimal placement of phasor measurement units for power system observability. IEEE Transactions on Power Systems, 23(3):1433-1440.

Chakrabarti S, Kyriakides E, and Eliades DG (2009). Placement of synchronized measurements for power system observability. IEEE Transactions on Power Delivery, 24(1): 12-9.

Gou B (2008). Generalized integer linear programming formulation for optimal PMU placement. IEEE Transactions on Power Systems, 23(3):1099-1104.

Jamuna K and Swarup KS (2012). Multi-objective biogeography based optimization for optimal PMU placement. Applied Soft Computing, 12(5): 1503-1510.

Kirkpatrick S, Gelatt CD, and Vecchi MP (1983). Optimization by simulated annealing. Science, 220(4598): 671-680.

Korres GN, Manousakis NM, Xygkis TC, and Lofberg J (2015). Optimal phasor measurement unit placement for numerical observability in the presence of conventional measurements using semi-definite programming. IET Generation Transmission and Distribution, 9(15):2427-2436.
Milosevic B and Begovic M (2003). Nondominated sorting genetic algorithm for optimal phasor measurement placement. IEEE Transactions on Power Systems, 18(1):69-75.

Mirjalili S, Mirjalili SM, and Yang XS (2014). Binary bat algorithm. Neural Computing and Applications, 25(3-4): 663-681.

Muller HH and Castro CA (2016). Genetic algorithm-based phasor measurement unit placement method considering observability and security criteria. IET Generation, Transmission and Distribution, 10(1): 270-280.

Phadke AG and Thorp JS (2008). Synchronized phasor measurements and their applications. Springer, New York, USA.

Phadke AG, Thorp JS, and Karimi KJ (1986). State estimation with phasor measurements. IEEE Transactions on Power Systems, 1(1): 233-238.

Rao RS, Narasimham SV, Raju MR, and Rao AS (2011). Optimal network reconfiguration of large-scale distribution system using harmony search algorithm. IEEE Transactions on Power Systems, 26(3): 1080-1088.

$\mathrm{Xu}$ J, Wen MH, Li VO, and Leung KC (2013). Optimal PMU placement for wide-area monitoring using chemical reaction optimization. In the IEEE PES Innovative Smart Grid Technologies, IEEE, Washington D.C., USA: 1-6. https://doi.org/10.1109/ISGT.2013.6497845

Yang XS (2010). A new met heuristic bat-inspired algorithm. In: Cruz C, González JR, Pelta DA, Krasnogor N, and Terrazas G (Eds.), Nature inspired cooperative strategies for optimization: 65-74. Springer, Berlin, Germany. 